\title{
Les mots du sida : néologie, obsolescence et fixation. Bilan terminologique de trois décennies d'épidémie et de recherche.
}

\author{
Gouttefangeas, Raphaëlle \\ Laboratoire de l'Arc Atlantique, Université de Pau \& des Pays de l'Adour \\ raphaelle.gouttefangeas@univ-pau.fr
}

L'objet de cette étude est d'envisager la terminologie du sida dans une perspective diachronique en prenant en compte les phénomènes de fixation, de figement et d'obsolescence.

Le sida, au-delà des perspectives synchroniques pertinentes dans le domaine scientifique, médical et plus particulièrement épidémiologique, se caractérise d'un point de vue linguistique par l'évolution des termes qui le désignent ou lui sont afférents (du nom de la maladie elle-même aux phraséologies connexes) au cours du processus de vulgarisation scientifique.

Je me propose ici d'étudier les phénomènes de création lexicale, d'obsolescence et de figement phraséologique dans une perspective à la fois descriptive et théorique, qui s'inscrit dans la lignée des principes posés par la théorie des portes de Maria Teresa Cabré ${ }^{1}$, laquelle situe la terminologie au confluent de plusieurs disciplines (sciences cognitives, linguistique, sciences du langage, sciences de la communication, sociolinguistique, etc.). L'étude de recommandations linguistiques concernant la communication sur le sida, émanant d'un organisme international, l'ONUSIDA, à travers un document de synthèse, le Guide de Terminologie de l'ONUSIDA (version révisée de 2011), permettra de mettre en perspective les principes extralinguistiques présidant aux choix lexicaux et surtout phraséologiques dans la terminologie du sida, et de s'interroger sur la veille néologique que le terminologue doit mettre en place dans ce domaine.

Ce travail sera conduit dans une perspective à la fois descriptive et théorique en tentant de voir comment le lexique du sida peut servir de prototype à une approche modulaire de la terminologie.

\section{Caractéristiques générales et spécificité de la terminologie du sida.}

Le choix de l'exemple terminologique du lexique du sida découle de plusieurs observations : la maladie apparue il y a un peu plus de 30 ans permet d'étudier, à travers un corpus abondant, la transition entre langue de spécialité et vulgarisation dans la presse grand public ; parce que cette maladie émergente est toujours incurable, elle continue à faire l'objet de recherches et de communications en permanence, de sorte que son lexique n'est que partiellement figé, ce qui permet au terminologue permet de continuer à étudier créations lexicales, nouvelles phraséologies et phénomènes d'obsolescence ${ }^{2}$; ces phénomènes nous amènent à prendre en compte les dimensions sociolinguistique (Gaudin, $1993^{3}$ ) et culturelle de cette terminologie, dans la mesure où les décisions lexicales dans ce domaine ne relèvent pas uniquement de facteurs scientifiques, mais reflètent également des présupposés et considérations sociologiques, sociolinguistiques, psychologiques et politiques - souvent exprimés par le biais de métaphores (Sontag, $1990^{4}$ ) sans oublier le rôle non négligeable des considérations normatives et prescriptives émanant d'organismes nationaux et internationauxMaladie inconnue avant les années 1980, le sida émerge d'abord comme un mal dont on ne connaît rien et dont le nom même reste sujet à controverse pendant un temps d'autant plus crucial que la maladie est mortelle et sans traitement. Comme le montrent clairement les récits des témoins directs de l'apparition de l'épidémie (patients, médecins, journalistes, militants) (Shilts, $1987^{5}$; Lestrade \& Pialoux, 2012 ${ }^{6}$ ), la découverte du sida va de pair avec la recherche de termes appropriés pour le dire. Le sida, tout comme une grande partie du lexique lié à des découvertes 
scientifiques et/ou médicales, oblige donc le terminologue à dévier de la perspective purement synchronique $^{7}$ telle que l'a développée Wüster (Van Campenhoudt, $2006^{8}$ ), d'autant plus qu'il est nécessaire de relayer cette information scientifique au plus vite auprès d'un public n'ayant non seulement aucune connaissance de la maladie en soi, mais aussi des connaissances plus que schématiques en matière d'épidémiologie ; ce public néanmoins est tout à fait réceptif au caractère anxiogène des informations diffusées au sujet du sida et avide de vulgarisation. Contrairement à des maladies plus anciennes, le sida, de par sa nature, génère un principe propre de création, d'évolution et d'obsolescence.

Ce processus terminologique d'obsolescence est manifeste dans les premières tentatives pour donner un nom au faisceau de pathologies repérées lors de l'observation clinique des premiers patients souffrant de cette maladie émergente aux Etats-Unis. Largement oublié aujourd'hui, l'acronyme GRID est effectivement le premier terme utilisé pour recouvrir l'ensemble de symptômes observés sur les premiers malades du sida; l'acronyme, rappelons-le, est formulé en anglais par des médecins américains à partir des observations qu'ils peuvent faire en 1981: comme tous les malades concernés sont des homosexuels masculins présentant des symptômes similaires (cancers, affections pulmonaires, sarcome de Kaposi, immunodépression), on choisit l'acronyme GRID qui signifie: « Gay-Related Immune Deficiency ». S'il y a très peu d'occurrences attestées de l'emploi de ce terme en français ${ }^{9}$, ses surnoms «gay plague »/ "gay cancer» vont rapidement prendre racine dans la terminologie relayée dans les médias français (Strazzula, $1993^{10}$ ), sous la phraséologie « cancer gay $»^{11}$. Dans la mesure où la maladie semble n'affecter qu'une portion de la population dont le comportement va à l'encontre d'un certain nombre de principes religieux, la création terminologique du début des années 1980 est empreinte de cette stigmatisation: l'équation [cancer homosexuel = punition divine] revient régulièrement dans les propos des journalistes (dans la presse écrite en particulier ${ }^{12}$ ), même si on peut douter de la validité d'une métaphore faisant du cancer une punition divine; en d'autres termes, le cancer, lorsqu'il affecte les homosexuels, est un châtiment, tandis que c'est une épreuve lorsqu'il s'attaque à la population considérée comme non déviante. La notion de groupe à risque se retrouve dans une autre dénomination ultérieure, «maladie des $4 \mathrm{H} »^{13}$ (Haïtiens, Homosexuels, Hémophiles, Héroïnomanes) qui elle aussi propose une indentification et une désignation du malade "type », et, d'une certaine manière, l'isole du reste de la population, considéré comme «saine» à plus d'un titre. Les connotations très négatives teintées d'homophobie du terme "peste» (Sontag, 1990 ${ }^{14}$ ) conjuguées à l'inexactitude des observations médicales (le sida n'affecte pas que les homosexuels ou les quatre groupes définis par la maladie des $4 \mathrm{H}$ ) expliquent largement l'obsolescence de ces termes dans la terminologie contemporaine.

Pour remplacer ces dénominations dont le corps médical et la presse ne peuvent que percevoir la dimension négative, le choix ultérieur du terme «S.I.D.A.» (acronyme qui deviendra rapidement normalisé: S.I.D.A., puis SIDA, puis Sida, puis enfin sida) correspond à un choix terminologique conscient qui se veut consensuel : décrire l'état que provoque la maladie («syndrome ») plutôt que le groupe qu'elle affecte le plus au sein de la population. Certes, le terme «SIDA » correspond aussi à certains critères de dénomination courants dans le domaine médical, les acronymes permettant la concision et la simplification, ainsi qu'une mémorisation plus facile du nom des pathologies médicales, mais il sert surtout ici à imposer une norme internationale permettant aux chercheurs de communiquer et de faire progresser ensemble la recherche sur la maladie. Notons néanmoins les commentaires, par exemple, d'un spécialiste français de la maladie, Willy Rozenbaum, qui explique le choix d'un acronyme différent de l'anglais non par exception culturelle mais pour éviter les connotations trop positives qu'aurait eues le terme AIDS (phonétiquement proche de "aides») en français pour désigner une maladie mortelle: "AIDS avec sa consonance homonymique positive, sonnait bizarrement en français pour un syndrome tueur et nous voulions trouver une équivalence $\gg(\text { p. } 50)^{15}$. Le choix de ne pas prendre un terme perçu comme " positif» donne une idée de la dimension extralinguistique qui peut s'attacher au simple nom commun de la maladie.

Les polémiques entourant le choix d'un nom pour la maladie prennent un tour différent en 1983 avec la découverte du virus à l'origine du sida. Les démêlés légaux entre les équipes de chercheurs américains et français posent alors un problème terminologique assez peu courant, à savoir que deux acronymes distincts ont émergé, un de chaque côté de l'Atlantique, pour désigner le même virus (Seytre, $1993^{16}$ ). 
Cette guerre des brevets (Thiaudière, $2002^{17}$ ) aboutit d'un point de vue lexical, un fois la querelle médicale réglée, à ce qu'un autre terme s'impose dans les deux langues en 1986, soit plus de trois ans après la découverte de la maladie : «HIV » (Human Immunodeficiency Virus) en anglais ${ }^{18}$ et «VIH » (virus de l'immunodéficience humaine) en français. Ainsi, la guerre physiologique et légale qui se déroulait entre Robert Gallo ${ }^{19}$ et son LTLV-III, et Luc Montagnier ${ }^{20}$, qui propose quant à lui de nommer le virus LAVà partir des souches étudiées en France, se termine, d'un point de vue terminologique, par le choix commun d'un néologisme imposé avec sa traduction simultanée dans les deux langues.

Plusieurs phénomènes intéressants sont à noter ici. Tout d'abord, le fait que les deux sigles coexistent pendant plusieurs années en français, reliés par différents marqueurs typographiques : HIV-VIH ou VIH (HIV) ou encore VIH/HIV, ce qui permet de voir le lien entre l'anglais et le français dans le choix de l'acronyme. Deuxièmement, la conjonction entre le virus et la maladie sous la forme «HIV/AIDS » ou «HIV/SIDA », entre autres dans des phraséologies, ce qui laisse transparaître une relation d'équivalence entre le virus et la maladie pendant une longue période de temps, particulièrement dans l'imaginaire collectif.

L'aspect dynamique de la terminologie liée au sida correspond par la suite aux progrès de la recherche médicale. La maladie qui reste mortelle et incurable, est désormais nommée, mais le lexique qui lui correspond évolue au fil des découvertes. L'acronyme SIDA ne nécessite plus qu'on l'explique entre parenthèses ou qu'on le marque comme néologisme par des guillemets dans la presse, puis il perd ses majuscules et entre dans le dictionnaire sous la forme «sida » »; le sida se décline aujourd'hui en deux virus : (HIV1 et HIV2 et aussi, en français, VIH-1 et VIH-2) ${ }^{21}$.

\section{Un cas d'étude : terminologie prescriptive dans le guide de l'ONUSIDA}

La prise de conscience internationale de l'étendue de l'épidémie dans le milieu des années 1980 aboutit à la création d'organismes multinationaux ou supranationaux pour endiguer (à défaut de pouvoir éradiquer) la maladie, qui est devenue une "pandémie». Il est intéressant de remarquer que le besoin terminologique concernant le sida se fait très fortement sentir dans toutes les langues, l'épidémie mortelle se répand sur toute la planète, ce qui fait de la diffusion des informations, de la traduction et de la vulgarisation des termes en rapport avec le sida un enjeu parallèle à celui de la recherche médicale.

L'ONU, à travers son programme ONUSIDA, a fait de la lutte contre le sida une de ses priorités. Ainsi, le métadiscours terminologique (prescriptif, normatif, critique) accompagnant la construction du lexique du sida est particulièrement révélateur, car il démontre que la dimension sociocognitive est extrêmement prégnante dans ce domaine terminologique. La présente étude prend pour support un document de portée internationale récent, émanant d'un organisme supranational, l'ONUSIDA, document émis à destination des traducteurs mais aussi des locuteurs francophones (rédacteurs scientifiques, soignants, mais aussi malades, accompagnants, etc.) dans son Guide de Terminologie de 2007 révisé en $2011 .^{22}$ L'ONUSIDA déclare dès l'introduction:

Dans la mesure où le langage donne forme aux concepts et peut influencer les comportements, l'emploi réfléchi d'un langage approprié est de nature à renforcer la riposte mondiale au VIH.

Cette déclaration souligne à quel point la terminologie employée peut prendre une dimension non seulement psychologique, mais aussi sociologique. De la même façon, l'UNESCO, organisation chargée des programmes concernant l'éducation, la science et la culture au sein des Nations Unies, emploie les vocables «formulations contestables»/ «formulations recommandées» dans sa brochure en ligne intitulée « Recommandations de l'UNESCO pour la terminologie et la formulation de documents relatifs au VIH et au SIDA ». La raison évoquée est :

Le VIH et le SIDA appellent des réponses de chacun d'entre nous, tant sur le plan individuel que sur le plan institutionnel. Pour être formulées, ces réponses doivent être traduites en images, en paroles et en textes. ${ }^{23}$ 
L'UNESCO souligne particulièrement, dans les recommandations ci-dessus, la nécessité de choisir le lexique utilisé pour parler du sida non pas pour des questions scientifiques, mais en tenant compte de la dimension psychologique et sociologique du lexique sélectionné, décrivant la "stigmatisation » comme un «obstacle à la prévention» et proposant une «harmonisation de la terminologie » en prenant particulièrement en compte les "groupes les plus vulnérables ». La brochure établit un lien permanent entre contexte médical, choix des termes et connotations associées, ce qui repose sur le présupposé que choisir le mauvais terme contribue à la propagation de la maladie. Dans ce contexte, le terminologue ne peut que constater que le lexique considéré a perdu tout ou une partie de sa neutralité.

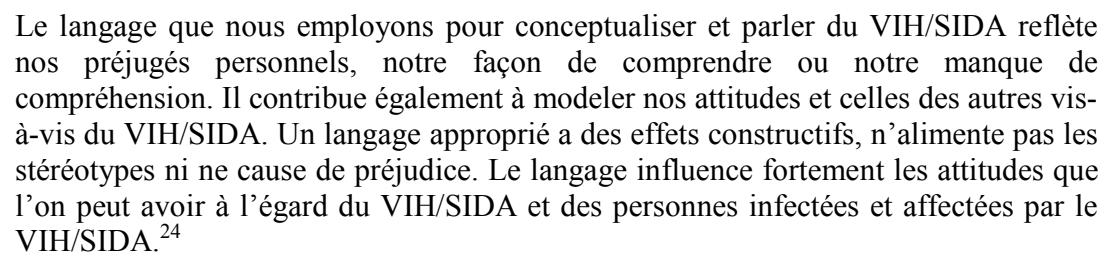

De la même façon, l'ONUSIDA se donne pour mission de dresser une liste aussi exhaustive que possible de ce qui peut ou ne peut pas se dire. Si l'on considère la formulation de ce guide, la mention entre parenthèses de l'expression « (à éviter) » derrière certains termes pousse le terminologue à réfléchir sur les critères d'exclusion: pour quelle(s) raison(s) doit-on éviter ces termes? Un examen des termes à ne plus employer fait également apparaître une liste de nouveaux termes recommandés pour les remplacer ; dans la mesure où ils sont couramment employés dans les traductions ou les articles de presse grand public, la répercussion de ces recommandations ur la vulgarisation de la connaissance scientifique est non négligeable.

La première motivation peut sembler médicale, l'ONUSIDA recommandant de ne pas confondre sida et VIH, c'est-à-dire bien identifier la maladie pour agir en conséquence :

$\mathrm{VIH} /$ sida

Utiliser le terme précis qui correspond au contexte pour éviter les confusions entre le $\mathrm{VIH}$ (un virus) et le sida (un syndrome clinique). ${ }^{25}$

Infecté(e) par le sida

On ne peut pas être infecté(e) par le sida, car ce n'est pas un agent infectieux. ${ }^{26}$

L'emploi de termes comme «virus », «syndrome» ou «agent infectieux » situe clairement cette recommandation dans un domaine médical, de façon à ce qu'une confusion lexicale ne génère pas d'erreurs de diagnostic. L'ONUSIDA se propose donc de rectifier des erreurs commises dans le cadre de la vulgarisation de termes médicaux et de leur diffusion dans la presse grand public, souvent par le biais de traductions. Autre exemple :

Taux de prévalence du VIH

Utiliser prévalence du VIH. Le terme taux implique le passage du temps et ne doit pas être utilisé en relation avec la prévalence. En revanche, il peut servir pour désigner l'incidence au fil du temps, par exemple 6 pour 100 de personnes/années. ${ }^{27}$

Néanmoins, la description des phraséologies à «préférer » ou à «éviter » réfère bien plus à la façon dont la maladie est perçue qu'à des préoccupations médicales descriptives. Prenons deux cas précis :

Victime du sida ou personne souffrant du sida.

Préférer personne vivant avec le VIH. Le mot victime a une connotation de passivité. $^{28}$

Ici, le caractère prescriptif de la recommandation - l'infinitif s'appuie sur une affirmation catégorique - a pour objet d'éviter la stigmatisation qu'engendrerait le choix d'une phraséologie alors que l'autre parait plus pertinente. Il s'avère donc ici que la terminologie recommandée, un euphémisme, se fonde sur une volonté de ne pas exclure ou isoler le malade en nommant simplement son état.

Le document poursuit, recommandant: 
Porteur, porteuse du sida (à éviter)

Ce terme n'est plus utilisé, car il est inexact, stigmatisant et blessant pour de nombreuses personnes vivant avec le VIH. ${ }^{29}$

Le critère de sélection marque fortement l'obsolescence du terme («n'est plus utilisé ») et l'explication fournie insiste sur la dimension sociologique du terme à proscrire, l'adjectif «stigmatisant » renforçant le sens de «blessant», ce qui confère au critère d'inexactitude une importance moindre. La phraséologie « population relais » est écartée, quant à elle, pour éviter de désigner des groupes plus susceptibles de contracter le VIH, et par conséquent, de définir des critères d'exclusion. Dans le même esprit, l'ONUSIDA propose d'éviter «lutte contre le sida ${ }^{30}$; les raisons évoquées ne sont pas non plus médicales mais prennent en compte l'effet psychologique de ce terme sur le lectorat:

Éviter les termes à connotation militaire tels que lutte, combat, campagne, guerres,
etc., sauf en cas de citation directe ou en raison du contexte précis d'un texte. [...] Les
alternatives possibles sont : riposte, prise en charge, mesures, initiative, actions,
efforts, programmes, etc. Ceci pour éviter, entre autres, un transfert de la lutte contre
le VIH à une lutte contre les personnes vivant avec le VIH. ${ }^{31}$

Soulignons au passage que l'on peut s'interroger sur le choix d'un terme comme « riposte » pour éviter des connotations « milliaires ». L’UNESCO fait bien référence par un acronyme, PLNS, au « programme national de lutte contre le sida », mais cette remarque de l'ONUSIDA est à mettre en perspective.

Par ailleurs, la définition que donne le dictionnaire Larousse du terme « lutte » laisse penser que ce terme serait tout à fait approprié à une utilisation dans le domaine épidémiologique et serait de surcroît chargé de connotations positives: «Ensemble des actions menées pour vaincre un mal: Lutte contre le cancer. $»^{32}$

Pourquoi donc réfuter le terme «lutte» ici ? Les termes de substitution proposés tendent à faire disparaître l'évocation directe de la maladie (faudrait-il parler, au lieu de lutte contre le sida, d'efforts pour la guérison?). Enfin, la raison donnée par l'organisme est encore celle d'une stigmatisation où le malade serait confondu avec la maladie, et la lutte contre cette maladie le prendrait pour cible.

Cette considération n'est pas sans rappeler certains débordements verbaux et créations lexicales assimilant le malade à la maladie. Dans le milieu des années 1980, le sida est de plus en plus perçu comme une menace pour l'ensemble de la population et dans ce contexte, ce n'est plus un type de malade en particulier mais le malade lui-même qui doit être isolé. Les compositions que proposent des hommes politiques, certes à la recherche de termes frappants sur un thème d'actualité (Favre, 1992), déplacent donc le registre terminologique médical dans un registre péjoratif et stigmatisant. Dire comme le fait Jean-Marie le Pen, sur Antenne2, dans l'émission L'Heure de Vérité du 6 mai $1987^{33}$, que « le sidaïque est une forme de lépreux » et suggérer qu'on le mette dans un "sidatorium », constitue une création néologique dont l'objectif n'est pas de nommer, mais de repérer, d'isoler et probablement de stigmatiser le malade (certains y voient un rapport inquiétant avec «judaïque » et «sanatorium », voire «crématorium »). Le terme «sidaïque » tombera donc dans l'obsolescence non en raison de son inexactitude scientifique, mais pour des raisons de prescription normative : l'employer est considéré comme une prise de position personnelle et peut-être même politique. Ainsi, le Dictionnaire de l'Académie française signale :

La forme sidaïque a été condamnée: Le préservatif plutôt que les mots qui tuent: sidaïque et sidatorium [établissement médical où seraient soignés en exclusivité les sidéens]. Il avait, en effet, suffi de ces deux expressions pour anéantir tous les efforts du petit César de la Trinité-sur-Mer (J.-M. Le Pen) pour acquérir une vraie respectabilité (L'Événement du Jeudi, 29 juill. 1987, p. 12) 34

Le guide publié par l'ONUSIDA met donc en exergue le rôle actif de la terminologie non seulement dans la vulgarisation et la compréhension du lexique spécialisé au sein de la population mais aussi le caractère polémique, politique, psychologique et aussi social des termes choisis, dont le simple choix peut véhiculer une dimension perçue comme «stigmatisante », particulièrement lorsque l'on compose des 
phraséologies : « malade du sida » en vient vite à être synonyme avec « grabataire contagieux », «porteur du virus » avec « danger ambulant » et « population à risque » avec « groupe à exclure ».

\title{
3 Veille néologique et terminologie du sida
}

La communication institutionnelle, donc, fournit un corpus extrêmement riche permettant d'engager une réflexion en profondeur sur une approche multidimensionnelle, modulaire, de la terminologie.

Le travail terminologique à poursuivre semble s'imposer au vu des nombreuses conférences, nationales et internationales, mettant en jeu à la fois l'étude des termes employés sur le terrain, leur réception par les populations cibles et l'analyse sociologique et culturelle que l'on peut effectuer. Citons à titre d'exemple les Actes de la Table ronde de l'UNESCO de novembre 2002 et ayant pour titre : "VIH/SIDA : stigmatisation et discrimination, une approche anthropologique $»^{35}$. Cette publication, se donne l'objectif suivant :

\begin{abstract}
Entendant la culture dans son sens le plus large - traditions, croyances, valeurs, structures familiales, rapports hommes/femmes, relations personnelles et sociales - ce projet encourage la pleine prise en compte des cultures des populations dans le développement des stratégies, projets et programmes de prévention du VIH/SIDA (p.i).
\end{abstract}

La réflexion terminologique, comme permet de le penser cette analyse, doit donc dans le cas précis du sida ne pas négliger la dimension socioculturelle des choix sémantiques et lexicaux effectués pour exprimer la maladie. L'impact de critères humains, affectifs, culturels, particulièrement, incite à une approche culturelle et diachronique continue de ce répertoire terminologique. Un autre exemple frappant est celui du document de travail à destination des professionnels de la Santé émis par l'INPES (Institut National de Prévention et d'Education à la Santé) intitulé Dépistage du VIH/SIDA chez la personne migrante/étrangère ${ }^{36}$. Ce document datant de 2004 recommande explicitement aux médecins et personnel médical : «Prudence sur la terminologie... » (p.4); on peut s'interroger au passage sur la signification de la ponctuation : faut-il lire : «prudence... ou gare aux conséquences»? Ou encore «prudence... et modération »? Au fond, les points de suspension laissent imaginer le pouvoir latent de la terminologie choisie dans ce domaine. Par ailleurs, le document fait état des difficultés terminologiques rencontrées par les malades ; tout en soulignant la nécessité d'avoir recours à, un interprète, il décrit des difficultés moins lexicales que psychologiques :

\begin{abstract}
Sur le registre émotionnel et symbolique, on enregistre des évocations morbides, anxiogènes, des représentations stigmatisantes des personnes contaminées, qui souvent incarnent la faute.

Il ressort des attitudes de mise à distance de la maladie, « c'est un peu tabou, honteux, on n'en parle pas » et des personnes à risque « je pense que si on discute avec quelqu'un, si on lui serre la main, il y a des risques... ». Sont décrits également des mécanismes de déni de l'existence de la maladie : « toutes les autres maladies, il y a des médicaments, mais là, il n'y a rien. Cette maladie là, je crois que ça n'existe pas ». (p.2)
\end{abstract}

Les concepts de «tabous », de « mise à distance », de « déni » incitent justement à la vigilance quant aux risques que comporte la vulgarisation de ce type de lexique. Remarquons néanmoins que l'intitulé de ce document illustre lui-même, d'une certaine façon, une logique d'exclusion.

Le Plan national de lutte contre le VIH/SIDA 2010/2014, repris dans un document ${ }^{37}$ émanant du Ministère français de la Santé et des Sports, a pour quatrième axe «Prise en charge sociale et lutte contre les discriminations » et propose sobrement de :

Favoriser les approches respectueuses des choix d'orientation sexuelle en intervenant auprès des professionnels sanitaires et sociaux en contact avec la population des femmes et hommes homo-bisexuel(le)s (p.65) 
Cette liste ne peut être exhaustive dans le cadre de cette communication, mais le fait que les institutions nationales et internationales s'interrogent et se réinterrogent en permanence sur ce que l'on peut dire ou ne pas dire concernant le sida oblige le terminologue à opérer une veille néologique rigoureuse dans ce domaine. L'épidémie ayant plus de trente ans, on peut relever la présence d'éléments de synchronie dynamique (Gaudin, $1993^{38}$ ) dans le lexique du sida, à savoir que chaque nouvelle création non seulement s'inscrit dans un processus continu de création en terminologie médicale, mais aussi s'articule sur la réception des termes créés à différentes époques par le grand public.

Première constatation : la progression et la généralisation de l'acronyme VIH (équivalent de l'anglais HIV, qui lui est d'ailleurs souvent adjoint sous la forme VIH/HIV). L'acronyme est repris in extenso dans les journaux et ne nécessite plus d'explication. Il apparaît de surcroît dans nombre de phraséologies reliées au sida : « virus du VIH», «vaccin contre le VIH», «dépistage du VIH », « infection par le VIH», « vivre avec le VIH », etc. Deuxième constatation : la nécessité d'établir une distinction entre VIH et sida dans les termes. L'ONUSIDA le rappelle dans le document analysé ci-dessus : les progrès médicaux permettent d'établir une terminologie qui distingue le virus (VIH) de l'état et des symptômes correspondant au stade déclaré de la maladie (sida). C'est une forme que l'on pourrait qualifier de chrysalide linguistique, puisque les deux acronymes désignent finalement deux états d'une même maladie. Ces distinctions s'accompagnent de considérations qui témoignent d'un degré avéré de vulgarisation scientifique : alors que la maladie était encore inconnue il y a un peu plus de 30 ans, tout marqueur typographique de néologisme (guillemets, explications entre parenthèses) a disparu dans la presse grand public lorsqu'on y fait référence.

Néanmoins, quelques éclaircissements et précisions sont encore nécessaires en raison de figements phraséologiques comme "virus du sida » et «virus (du) VIH », tous deux très courants mais incorrects scientifiquement. En effet, dans le cas du «virus du sida », la phraséologie ne tient pas compte du sens originel de l'acronyme (syndrome de l'immunodéficience acquise) : il n'existe pas de virus d'un syndrome, un syndrome se définissant comme un «ensemble de plusieurs symptômes ou signes en rapport avec un état pathologique donné et permettant, par leur groupement, d'orienter le diagnostic »" Quant à «virus du VIH », il s'agit d'un pléonasme ou plutôt d'une redondance implicite, le V de VIH indiquant le mot « virus ».

L'ONUSIDA insiste aussi sur la nécessité absolue de les distinguer, contrairement à ce qu'indique l'emploi très fréquent en langue anglaise de HIV/AIDS, la barre oblique pouvant être interprétée comme VIH et/ou SIDA, comme si aucune différence médicale n'existait entre les deux:

Il est préférable d'utiliser le terme qui correspond spécifiquement au contexte.

Par exemple : personnes vivant avec le VIH [...] diagnostic de sida [...]. Les termes épidémie de VIH et épidémie de sida sont tous deux acceptables, mais le premier est plus général. (p.14) ${ }^{40}$

On remarque à l'occasion que ce sont les phraséologies qui mettent en lumière les différences d'emploi entre les deux termes.

Des néologismes ont été formés pour désigner de nouvelles spécialisations médicales : " sidologue », « sidénologue », alors que la maladie était au départ étudiée par un ensemble de chercheurs de disciplines connexes, entre autres les épidémiologistes, les cancérologues, etc. (Lestrade, Pialoux, 1997). Sur le modèle du « kremlinologue », terme né de l'étude des bouleversements politiques affectant l'URSS, le sidologue apparaît pour désigner le spécialiste de cette nouvelle maladie.

Spécialiste du sida. Au congrès qui a réuni fin juin à Paris plus de 2000 sidologues (...), le ton n'a pas été à l'optimisme béat (...). Le SIDA est un casse-tête (L'Est Républicain, 31 juillet 1986, p. 32, col. 2). La transmission hétérosexuelle du SIDA est maintenant parfaitement démontrée malgré les combats d'arrière-garde de certains

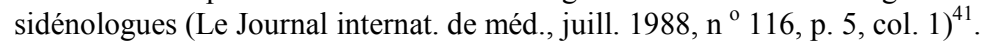

D'autres concernent les progrès scientifiques qui permettent aux malades de prolonger leur vie, comme la « trithérapie » (1996, traitements combinés) devenue aujourd'hui «polythérapie » ou « multithérapie », qui permet une «quasi-guérison » ou plutôt une "rémission persistante »" ${ }^{42}$. Dans la même ligne de 
recherche et le même registre que d'autres maladies incurables, l'hépatite B ou la maladie de Parkinson par exemple, des phraséologies comme « vaccin curatif contre le VIH » ou « vaccin thérapeutique contre le VIH » ont été formées. De ce fait, de nouveaux termes correspondant aux nouvelles réalités entourant le sida font leur apparition dans la langue commune, et témoignent d'une sorte de «normalisation de la maladie $»^{43}$

La difficulté, comme nous l'avons vu, à nommer le malade atteint du sida sans le stigmatiser conduit à récuser aujourd'hui des termes comme «sidatique », ou " sidéen ». Cependant, la définition de substituts acceptables par le Dictionnaire de l'Académie française reste ambiguë :

Sidéen, de par sa constr., semblerait mieux convenir à tout malade atteint d'un syndrome immuno-déficitaire, quelle que soit son origine (virale ou non), tandis que sidatique conviendrait mieux aux malades atteints de syndrome immuno-déficitaire acquis d'origine virale, c'est-à-dire du sida ${ }^{44}$.

En somme, le « sidéen » se distinguerait du « sidatique » parce qu'il ne souffrirait pas (encore) du sida.

Par ailleurs, la subjectivité attachée à ces choix est clairement perceptible au vu de l'émotion suscitée encore récemment (juin 2012) par l'emploi du très connoté «sidaïque », probablement par maladresse, sur le site de France 5 dans la description d'un documentaire ${ }^{45}$. Devant l'indignation générale, le site retire le terme «sidaïque » et le remplace par «porteur du VIH» dans la journée. C'est encore un choix malheureux si l'on se réfère à la brochure de l'ONUSIDA, puisqu'on peut y lire (cf. ci-dessus) :

Porteur, porteuse du sida (à éviter). Ce terme n'est plus utilisé, car il est inexact, stigmatisant et blessant pour de nombreuses personnes vivant avec le VIH. (p.6) ${ }^{46}$.

Que faut-il donc dire ? Il est intéressant de souligner la phraséologie «personne vivant avec le VIH » (ou, plus récemment, sous forme acronymique, PVVIH) présentée dans cette citation, ainsi que sa variante, " personne vivant avec le sida », car leur choix tend à affaiblir les connotations liées au mot sida, comme « incurable », «mortel», « létal », « fatidique », l'emploi de «verdict» pour « diagnostic», etc. En somme, la terminologie imposée (depuis l'anglais) propose de «vivre avec le sida » plutôt que d'en mourir. Ici, la dimension culturelle de la terminologie est très marquée: le terme "personne » peut s'appliquer aussi bien à un homme qu'à une femme, ce qui permet de ne pas viser les hommes homosexuels. Le choix du gérondif « vivant», calqué sur l'anglais «living », ainsi préféré à une relative ou à un seul terme, semble étrange dans le cadre d'un syntagme nominal, mais il permet toutefois d'attirer l'attention sur le fait que l'on peut être vivant et atteint du sida. L'ensemble du syntagme permet aussi de s'interroger sur la façon dont on vit avec le sida et non d'insister sur la contagion (en remplacement de « malade du sida » ou « personne atteinte du sida »). Il s'agit donc, à mon sens, d'une création/ adaptation néologique intéressante en français, dans le mesure où elle est unique (on ne parle pas de "personne vivant avec le diabète ou une quelconque autre maladie) et où elle permet un commentaire sémantique allant bien au-delà de la formulation pure et simple.

Cette formation sert elle-même de support à d'autres néologismes. La personne vivant avec le sida ou le VIH, devient, encore une fois sur le modèle anglais, " aids survivor », un « survivant du sida ». Citons ici en exemple le récent témoignage de Jean-Luc Romero dans le Dauphiné Libéré du 02/12/2012 $2^{47}$, ainsi que la une de nombreux journaux à l'occasion des 30 ans d'épidémie. Le Journal du Dimanche du 2 décembre 2012 titre : «À l'écoute des survivants du sida ». Comparativement aux «survivants à long terme » qui «[...] après plus de dix ans d'infection par le VIH, ne développent pas le sida.»" ${ }^{48}$, la phraséologie «survivants du sida » est surtout à considérer dans un contexte plus militant, comme la parole de ceux qui témoignent de leur résilience face à l'épidémie.

On distingue de fait de nouvelles catégories au sein de la population qui illustrent la durée de l'épidémie, les avancées médicales et l'émergence de nouveaux concepts afférents. Si les premiers malades, homosexuels, mal ou peu soignés, mouraient dans l'ensemble très vite, les personnes vivant avec le VIH aujourd'hui appartiennent à toutes les classes d'âges. Parallèlement aux «survivants », de nouveaux termes sont apparus pour décrire ceux que l'on appelait autrefois les «malades du sida» ou leur traitement. On parle ainsi, sous forme acronymique, de PTME, soit « Prévention de la Transmission de la 
Mère à l'Enfant »: il s'agit d'une thérapie visant à permettre que l'enfant d'une mère séropositive soit séronégatif à la naissance, c'est-dire en quelque sorte «guéri » du virus HIV. La dénomination choisie pour le traitement, tant dans la presse scientifique que dans les articles de vulgarisation destinés au grand public, gomme en quelque sort le lien avec la maladie, qui n'apparaît plus en toutes lettres (la «transmission» pourrait concerner n'importe quelle maladie infectieuse ou virale). Le choix de l'acronyme PTME ne fait que renforcer cet effet, dans la mesure où il tend à remplacer quasiment dès la mise en place la démnomination initiale de ce protocole thérapeutique sans avoir fait l'objet d'une phase de lexicalisation.

Le terme de «sérodiscordance» a également commencé à être employé. Il a pour racine les termes « séropositif» et "séronégatif», utilisés pour référer à l'état sérologique d'une personne qui a été dépistée comme atteinte du VIH. Ce terme se retrouve souvent dans la phraséologie «couple sérodiscordant » ou « sérodifférent» (aussi appelés couples «paradoxaux » ${ }^{49}$ sur le site de l'Inserm), pour référer à un couple ayant des statuts sérologiques opposés et fait l'objet de nombreuses discussions sur la prévention et la contamination. Les compositions « séro-discordant » et « sérodifférent » font d'ailleurs apparaître un phénomène linguistique intéressant. Leur formation repose sur «séropositif» (ou son antonyme, "séronégatif ») défini comme suit: "Qui donne une réaction positive à un ou plusieurs tests sérologiques ».

Ces termes sont donc des emprunts au domaine médical général surtout employés aujourd'hui en vulgarisation scientifique dans un sens lié au sida. Ils sont en effet composés à l'aide du formant SÉRO(du latin serum : « liquide séreux ») qui entre dans la composition de nombreux autres termes appartenant aux domaines de la biologie et de la médecine ne concernant pas le $s i d a^{50}$. La souplesse sémantique que l'on peut constater ici est en fait un glissement de sens: " sérodifférent » ou « séro-discordant » signifient plus que la somme de leurs signifiés et SERO-devient synonyme de «porteur ou non du virus du VIH ». Quant à «séropositif», bien qu'il puisse être utilisé de façon plus générale en biologie et en épidémiologie, il est devenu synonyme de «positif au virus du VIH », à tel point que l'apocope « séropo » est entré dans la langue courante, particulièrement sur Internet (sur les sites et forums destinés aux malades), mais aussi dans la presse grand public, comme le démontrent les titres d'articles suivants : «Afro, toxico et séropo » (Libération du 12 mai $2003^{51}$ ); « Sida : finalement, c'est plus dur d'être séropo en 2013 qu'il y a vingt ans» (Le Nouvel observateur du 13 septembre 2013 ${ }^{52}$ ), ou encore dans les émissions de radio : « La femme séropo qui voulait avoir un enfant » (france inter, émission diffusée le 30 mars $2012^{53}$ ). Son contraire, «séroneg», a un quasi statut d'hapax, ne figurant que sur des pages personnelles ou divers forums sur Internet. Cette observation peut également s'appliquer à d'autres termes qui ont aujourd'hui une dimension sémantique fortement connotée par l'épidémie de sida, comme " dépistage », " séroprévalence », " multithérapie », "charge virale », et se comportent plus ou moins comme des néologismes de sens. On peut donc souligner l'intérêt pour le terminologue d'être attentif non seulement aux mots nouveaux créés pour décrire l'évolution de l'épidémie, mais aussi aux répercussions linguistiques que peut avoir l'utilisation du terme sida sur certains termes courants dont le sens se fige dans un registre spécialisé.

Nous pouvons remarquer de nouveaux exemples issus de la presse très récente et nous interroger sur les orientations futures à donner à cette veille terminologique. Libération a publié le 10 mars 2014 un article dont le titre est «Sida : séropositifs, bien traités et non contaminants » ${ }^{54}$ pour parler d'avancées dans la prise en charge des personnes vivant avec le VIH. Le terme qui retient notre attention ici est bien entendu le syntagme «non contaminants ». Cet article fait écho à des publications précédentes dans le même journal, d'Eric Favereau, qui parlait déjà en 2011 de «résistants au sida ». Cependant, le terme "résistants" est en français chargé de connotations historiques et socioculturelles le plus souvent positives (combattant, courageux, face à l'oppression, etc.). Ici, la présence du marqueur du pluriel et de la négation permet, a contrario, de se représenter mentalement une population « contaminante », en lieu et place d'une population «contagieuse». Or, quel est le domaine d'application habituel du terme « contaminant »? Si le Trésor Informatisé de la langue française ne donne aucune définition de ce terme comme nom commun, il apparaît cependant fréquemment dans des dictionnaires de spécialité, sous la forme d'un nom commun, défini comme suit par exemple sur Aquaportail, site consacré à l'aquariophilie: 


\begin{abstract}
Désigne un élément ou une substance contenu [sic] dans l'environnement (air, eau, sédiments, etc.) en quantité anormale (c'est à dire non naturelle). Un excès de contaminants peut aboutir à une contamination.
\end{abstract}

De la même façon, le site du gouvernement canadien pour la protection de l'environnement, Environnement Canada, donne la défintion suivante pour le même terme : "Toute substance ou matière physique, chimique, biologique ou radiologique qui a un effet négatif sur l'atmosphère, l'eau ou le sol. $»^{55}$ Parmi les phraséologies relevées dans la presse récente, on peut signaler «contaminant chimique », le terme (un anglicisme avéré sur "contaminant»), dans des contextes d'écologie et de lutte contre la pollution et peut même référer à l'irradiation nucléaire ; dans la même tonalité, on relève, « contaminant atmosphérique », « présence de contaminants. », « lutte contre les contaminants », etc. Par ailleurs, son antonyme, "décontaminant ", se rencontre dans des contextes bactériologiques (par exemple, pour le produit utilisé avant le nettoyage d'un instrument médico-chirurgical souillé) ou chimiques (liquide utilisé pour annuler les effets de gaz lacrymogènes, de substances radioactives, etc.). Parler de «non contaminant » donne ainsi l'impression que le patient a subi une décontamination. Nous nous trouvons donc à nouveau devant une terminologie répondant à une logique d'exclusion : le terme «contaminant » (que rapporte le journaliste plutôt qu'il ne le choisit) déshumanise le malade, le comparant implicitement à un produit, un objet, une chose ; le terme « contaminant », employé comme adjectif, pour désigner des malades, remet en exergue leur rôle infectieux au sein de la population. Pourquoi ce choix ? Le "non contaminant» répond probablement à la double volonté d'annoncer un progrès dans le traitement de l'épidémie et de respecter cependant la vérité scientifique. L'article regorge de termes comme 《 avancées », « progrès » " bouleversements » mais les met à distance en ajoutant : "progrès pas à pas » ou « loin des grands bouleversements»; quant aux avancées, le journaliste insiste sur leur véracité : elles sont qualifiées de « réelles », tout comme les confirmations sont «essentielles». La question qui se dessine derrière ces choix lexicaux semble être : trente ans après le début de l'épidémie, comment entretenir l'intérêt et l'espoir? Il n'y a à ce jour ni traitement ni vaccin qui puisse éradiquer le sida. A défaut, le patient devient en quelque sorte un «porteur sain»; mais dans le cas du sida, le journaliste éprouve le besoin de créer un syntagme spécifique, ce qui une fois de plus illustre le caractère très inhabituel de la terminologie du sida : parler du «porteur sain » d'un « virus mortel » ressemble toujours à un oxymore. La conclusion est plus claire : « un séropositif bien traité n'est plus contaminant. 》 La terminologie médicale elle-même a recours à un néologisme de sens pour refléter le nouveau paradigme de la maladie : celui de patients qui vont parvenir à survivre : le terme " contrôleurs à long terme ", utilisé entre autres par l'Institut Pasteur, évacue le nom de la maladie ou même le statut de "patient»: le "contrôleur» est le patient bénéficiant d'une "rémission fonctionnelle » (porteur du virus mais asymptomatique), celui qui donc "contrôle » sa maladie. Néanmoins, la dimension métaphorique de ce terme permet de faire du survivant un témoin, un repère et un co-évaluateur de la progression de la maladie, dans une version nettement moins péjorative de la survie que le terme «contaminant». En attendant qu'un néologisme désigne celui ou celle qui sera guéri du sida, que ce soit par "vaccination thérapeutique » ou thérapie génique, et que le virus lui-même soit mis à contribution pour de plus nobles fonctions, comme la guérison de la leucémie. On peut s'attendre dans tous ces cas à un déluge de néologismes se prêtant à une analyse terminologique à de multiples niveaux.

Les exemples relevés récemment, à l'occasion des nombreux bilans de l'épidémie de sida au bout de trente ans, renforcent la perception modulaire que l'on peut proposer de la terminologie du sida.

Si certains termes sont tombés dans l'obsolescence, force est de constater, comme nous l'avons démontré, qu'il ne s'agit pas seulement de vulgariser de façon synchronique les progrès médicaux que reflète le lexique spécialisé de l'épidémiologie.

L'étude des termes de substitution, et plus particulièrement des phraséologies préconisées à la place d'autres expressions à proscrire, et ce dans un contexte de communication nationale et internationale, révèle l'aspect dynamique de ce lexique et la part d'éléments extralinguistiques entrant dans l'emploi de cette terminologie. 
Suite à ces constatations, la nécessité d'une veille terminologique, visant à mesurer l'impact de ces évolutions et recommandations sur les locuteurs francophones par le biais d'enquêtes linguistiques, me semble nécessaire pour établir la validité et la réception, ainsi que l'évolution des perceptions, parmi des locuteurs natifs francophones de tous horizons, des malades aux spécialistes, et de toutes classes d'âge ${ }^{56}$.

\section{Références bibliographiques}

\section{Ouvrages}

Cabre, M.T. et Sager, J.C. (1999). Terminology: Theory, Methods, and Applications. Amsterdam : John Benjamins Publishing Company.

Depecker L. (2005). « Contribution de la terminologie à la linguistique ». Langages, 39e année, $\mathrm{n}^{\circ} 157, \mathrm{La}$ terminologie : nature et enjeux. p. 6-13.

Desmet, I. (2007). « Terminologie, culture et société. Éléments pour une théorie variationniste de la terminologie et des langues de spécialité ». Cahiers du Rifal, $n^{\circ} 26$.

Dijker, A. (1998). « Aspects socio-psychologiques de la stigmatisation des personnes atteintes ». Le SIDA en Europe, nouveaux enjeux pour les sciences sociales : 2e conférence européenne sur les méthodes et les résultats des recherches en sciences sociales sur le SIDA. Paris : Agence Nationale de Recherches sur le Sida (éditeur), collection Sciences sociales et sida, p.125-132.

Dury P. et Picton A. (2009). « Terminologie et diachronie : vers une réconciliation théorique et méthodologique ?». Revue française de linguistique appliquée 2009/2 (Vol. XIV).

Gaudin F. (1993). Pour une socioterminologie. Des problèmes sémantiques aux pratiques institutionnelles. Rouen: Presse universitaires de Rouen.

Handman, M.-H. (1997). «La stigmatisation des femmes à travers les représentations du sida véhiculées par les médias ». Paris : Transcriptases n52. p.12-13.

Humbley, J. (2009). «Présentation. Terminologie : orientations actuelles ». Revue française de linguistique appliquée 2/2009 (Vol. XIV), p. 5-8.

Lestrade, D. et Pialoux G. (2012). Sida 2.0. Regards croisés sur 30 ans d'une pandémie. Paris : Fleuve Noir Docs.

L'homme M.-C.'2004). La terminologie : principes et techniques, Québec : Les Presses de l'Université de Montréal.

Rey, Alain (1970) La lexicologie. Lectures. Paris ; Klincksieck.

Mortureux, M.-F. (1997). La lexicologie entre langue et discours. Paris : Sedes.

Rozembaum, W. (1999) La vie est une maladie mortelle sexuellement transmissible. Paris: Stock.

Seytre B. (1993). Sida : les secrets d'une polémique. Paris : Presses universitaires de France.

Sontag S. (1990). Illness as Metaphor and Aids and its Metaphors. New York : Picador.

Manuel Ureña J., Faber P. (2013). « The world meets the body: Sociocultural aspects of terminological metaphor ». Proceedings of the 37th Annual Meeting of the Berkeley Linguistics Society. http://elanguage.net/journals/bls/article/view/3613.

Sablayrolles, J.-F. (éd.) ( 2003). L'innovation lexicale. Paris : Honoré Champion.

Strazzulla J. (1993). Le sida. 1981-1985.Les débuts d’une pandémie. Paris : La documentation française.

Temmerman, R. (2000). «Une théorie réaliste de la terminologie : le sociocognitivisme ». Terminologies Nouvelles, $n^{\circ} 21$, p. $58-64$.

Thiaudière C. (2002) Sociologie du sida. Paris : La Découverte.

Van Campenhoudt, M. (2006). «Que nous reste-t-il d'Eugen Wüster? ». Colloque international «Eugen Wüster et la terminologie de l'École de Vienne », Université de Paris 7, 3 et 4 février 2006.

http://www.termisti.refer.org/wuster.pdf. 


\section{Brochures émanant d'organismes officiels consultables en ligne}

INPES. (2004) « Dépistage du VIH/SIDA chez la personne étrangère ». http://www.inpes.sante.fr/CFESBases/catalogue/pdf/742.pdf

Ministère des Affaires Etrangères. (2007). "L'action de la France dans la lutte contre le sida ». http://www.ambafrance-at.org/IMG/pdf/sida.pdf

Ministère de la Santé. (2010). «Plan_national_lutte_contre_le_VIH-SIDA_et_les_IST_2010-2014». http://www.sante.gouv.fr/IMG/pdf/plan national lutte contre le VIH-SIDA et les IST 2010-2014.pdf

OMS. (2005). Directives conjointes OIT/OMS sur les services de santé et le VIH/SIDA. http://www.who.int/hiv/pub/prev_care/who_ilo_guidelines_fr.pdf

ONUSIDA. (2011). Guide de Terminologie de l'ONUSIDA. http://www.unaids.org/en/media/unaids/contentassets/documents/unaidspublication/2011/JC2118 terminologyguidelines fr.pdf.

UNESCO. (2008). Recommandations de l'UNESCO pour la terminologie et la formulation de documents relatifs au VIH et au SIDA. http://unesdoc.unesco.org/images/0014/001447/144725f.pdf. Consulté en ligne le 11/12/2013.

"VIH/SIDA : stigmatisation et discrimination, une approche anthropologique ». Paris : Actes de la table ronde organisée le 29 novembre 2002 à l'UNESCO. http://unesdoc.unesco.org/images/0013/001307/130756f.pdf. Consulté en ligne le 11/02/2013.

WHO. (2009). «Global health sector strategy on hiv/aids 2011-2015». http://whqlibdoc.who.int/publications/2011/9789241501651 eng.pdf?ua=1

${ }^{1}$ Cabre, M.T. et Sager, J.C. (1999). Terminology: Theory, Methods, and Applications. Amsterdam : John Benjamins Publishing Company.

${ }^{2}$ L'obsolescence, telle que la définit le site canadien Termium Plus ${ }^{\circledR}$, est un «terme littéraire et vieilli, il se dit, non exclusivement mais surtout, de ce qui se rapporte à la langue, au vocabulaire, à la grammaire. Est obsolète ce qui est dépassé, suranné, désuet, vieillot, bref, ce qui ne fait plus partie des normes actuelles du langage".Termium +, http://www.btb.termiumplus.gc.ca/tpv2guides/guides/juridi/index-

fra.html?lang=fra\&lettr=indx catlog o\&page=9mpnTBAVB-iE.html. Ce concept est repris aussi sous la dénomination "mort lexicale "(Grzega, 2012).

${ }^{3}$ Gaudin F. (1993). Pour une socioterminologie. Des problèmes sémantiques aux pratiques institutionnelles. Rouen : Presse universitaires de Rouen.

${ }^{4}$ Sontag S. (1990). Illness as Metaphor and Aids and its Metaphors. New York : Picador.

${ }^{5}$ Shilts R. (1987). And the Band Played On. Politics, People, and the AIDS epidemic. Middlesex : Penguin Books.

${ }^{6}$ Lestrade, D. et Pialoux G. (2012). Sida 2.0. Regards croisés sur 30 ans d'une pandémie. Paris : Fleuve Noir Docs.

${ }^{7}$ «Les remises en perspective et les remises en cause des idées-forces de Wüster sont, certes, souvent venues des sciences du langage, mais il est intéressant de noter que tous les chercheurs qui ont marqué la discipline au cours des dernières années semblent avoir voulu inventer des sous-disciplines : on dispose désormais d'une socioterminologie, d'une ontoterminologie, d'une terminologie sociocognitive, d'une terminologie textuelle, d'une pragmatoterminologie [...] 》 Van Campenhoudt M. (2006). "Que nous reste-t-il d'Eugen Wüster ? », Colloque international Eugen Wüster et la terminologie de l'École de Vienne, Université de Paris 7, 3 et 4 février 2006. http://www.termisti.refer.org/wuster.pdf

${ }^{8}$ Van Campenhoudt, M. (2006). «Que nous reste-t-il d'Eugen Wüster? ». Colloque international «Eugen Wüster et la terminologie de l'École de Vienne », Université de Paris 7, 3 et 4 février 2006. http://www.termisti.refer.org/wuster.pdf.

${ }^{9}$ A cette époque où Internet n'existe pas encore, les informations circulent d'une part par le bouche-à-oreille (via des personnes ayant voyagé aux Etats-Unis), d'autre part par la presse dite spécialisée, en particulier dans le Gai Pied, journal d'information homosexuel, qui titre: "Amour à Risque» dès septembre 1981. Voir aussi la une de Libération du 17 mai 1983 qui titre : " 'cancer gay', la contagion par la le sang. » 


\footnotetext{
${ }^{10}$ Strazzulla J. (1993). Le sida. 1981-1985.Les débuts d'une pandémie. Paris : La documentation française.

${ }^{11}$ Notons à cette occasion l'apparition du terme « gay » dans la presse grand public, dont l'utilisation va croître avec la diffusion de l'information sur la maladie. L'homosexualité, à peine dépénalisée avec la présidence de François Mitterrand, va alors faire l'objet d'une diffusion médiatique et terminologique croissante au fur et à mesure que la maladie attire l'attention médiatique.
}

${ }^{12}$ Voir, par exemple, Le Matin de Paris du 2 janvier 1982.

${ }^{13}$ Voire « maladie des $5 \mathrm{H}$ » si l'on inclut « hooker » : prostituée en anglais, mais cette référence n'est pas utilisable en français.

${ }^{14}$ Sontag S. (1990). Illness as Metaphor and Aids and its Metaphors. New York : Picador,

${ }^{15}$ Rozembaum, W. (1999) La vie est une maladie mortelle sexuellement transmissible. Paris: Stock.

${ }^{16}$ Seytre B. (1993). Sida : les secrets d'une polémique. Paris : Presses universitaires de France.

${ }^{17}$ Thiaudière C. (2002) Sociologie du sida. Paris : La Découverte.

18 Imposé dans la revue médicale MMWR (Morbidity and Mortality Weekly Reports), publication du centre d'épidémiologie américain, le CDC (Center for Disease Control and Prevention,) concernant les recommandations et données collectées par les agences de santé américaines.

${ }^{19}$ Robert GALLO, né en 1937, chercheur américain en immunologie et virologie, spécialiste des rétrovirus, directeur de l'Institute of Human Virology, Baltimore, Maryland, USA.

${ }^{20}$ Luc Montagnier, né en 1932, virologue français, colauréat avec Françoise Barré-Sinoussi du Prix Nobel de physiologie ou médecine de 2008 pour leur découverte en 1983 du VIH, le virus responsable du SIDA.

${ }^{21}$ Le VIH est un rétrovirus à la variabilité génétique très importante qui se décline en deux sous-types, 1 et 2 , et à l'intérieur, en de nombreux sous-groupes, chaque patient présentant quasiment une souche unique. La découverte du VIH-2 correspond à un VIH considéré comme moins contagieux et identifié parmi les populations d'Afrique de l'Ouest. Cette taxonomie est bien entendu appelée à évoluer et à produire d'autres néologismes médicaux au fur et à mesure des découvertes épidémiologiques dans ce domaine.

${ }^{22}$ Guide de Terminologie de l'ONUSIDA.,

http://www.unaids.org/en/media/unaids/contentassets/documents/unaidspublication/2011/JC2118 terminologyguidelines_fr.pdf. Consulté en ligne le 08/12/2013.

${ }^{23}$ Recommandations de l'UNESCO pour la terminologie et la formulation de documents relatifs au VIH et au SIDA. http://unesdoc.unesco.org/images/0014/001447/144725f.pdf. Consulté en ligne le 11/12/2013.

${ }^{24}$ Ibid.

${ }^{25}$ Guide de Terminologie de l'ONUSIDA.

http://www.unaids.org/en/media/unaids/contentassets/documents/unaidspublication/2011/JC2118 terminologyguidelines fr.pdf. Consulté en ligne le 08/12/2013.

${ }^{26}$ Ibid. p.5

${ }^{27}$ Ibid. p.5

${ }^{28}$ Ibid. p.5

${ }^{29}$ Ibid. p. 6.

${ }^{30}$ Ibid. p. 6

${ }^{31}$ Ibid.p.6

${ }^{32}$ Larousse.fr, http://www.larousse.fr/dictionnaires/francais/lutte/48139. Consulté en ligne le 11/12/2013.

${ }^{33}$ Ina.fr. «Le Pen sur le sida (le sidaïque est une espèce de lépreux) ». http://www.ina.fr/video/I00005232/suite-lepen-sur-le-sida-le-sidaique-est-une-espece-de-lepreux-video.html. Consulté en ligne le 11/12/2013. 
34 Atilf, http://atilf.atilf.fr/dendien/scripts/tlfiv5/visusel.exe?35; $=3208697130 ; \mathrm{r}=3 ; \mathrm{nat}=; \mathrm{sol}=0$. Consulté en ligne le $12 / 12 / 2013$.

35 «VIH/SIDA : stigmatisation et discrimination, une approche anthropologique ». Paris : Actes de la table ronde organisée le 29 novembre 2002 à l'UNESCO. http://unesdoc.unesco.org/images/0013/001307/130756f.pdf.

${ }^{36}$ INPES. « Dépistage du VIH/SIDA chez la personne étrangère ». http://www.inpes.sante.fr/CFESBases/catalogue/pdf/742.pdf

37 Ministère de la Santé. «Plan_national_lutte_contre_le_VIH-SIDA_et_les_IST_2010-2014». http://www.sante.gouv.fr/IMG/pdf/plan_national_lutte_contre_le_VIH-SIDA_et_les_IST_2010-2014.pdf

${ }^{38}$ Gaudin F. (1993) Pour une socioterminologie. Des problèmes sémantiques aux pratiques institutionnelles. Rouen: Presse universitaires de Rouen.

${ }^{39}$ Larousse.fr, http://www.larousse.fr/dictionnaires/francais/syndrome/76170. Consulté en ligne le 12/12/2013.

${ }^{40}$ Guide de Terminologie de l'ONUSIDA.

http://www.unaids.org/en/media/unaids/contentassets/documents/unaidspublication/2011/JC2118 terminologyguidelines_fr.pdf. Consulté en ligne le 08/12/2013.

${ }^{41}$ Atilf. http://atilf.atilf.fr/dendien/scripts/tlfiv5/visusel.exe?35; $\mathrm{s}=3208697130 ; \mathrm{r}=3 ; \mathrm{nat}=; \mathrm{sol}=0 ;$. Consulté en ligne le 07/12/2013.

42 MidiLibre.fr. "Sida : après 30 ans de recherche, la "quasi-guérison" à l'ordre du jour ». 21/05/2013, http://www.midilibre.fr/2013/05/21/sida-apres-30-ans-de-recherche-la-quasi-guerison-a-1-ordre-du-jour,700843.php. Consulté en ligne le 12/12/2013.

${ }^{43}$ Favereau, E. «Sida : un traitement pour tout le monde, tout de suite». Libération.fr. 30 septembre 2013, http://www.liberation.fr/societe/2013/09/30/sida-un-traitement-pour-tout-le-monde-tout-de-suite 935861. Consulté en ligne le $09 / 12 / 2013$.

${ }^{44}$ Atilf, http://atilf.atilf.fr/dendien/scripts/tlfiv5/visusel.exe?35;s=3208697130;r=3;nat=;sol=0; consulté en ligne le $11 / 12 / 2013$.

${ }^{45}$ Loncol, J.et M. (2012). «Un homme s'accuse sous le regard des experts ». Le Monde en face, France 5, 12 juin 2012.

${ }^{46}$ Guide de Terminologie de l'ONUSIDA.

http://www.unaids.org/en/media/unaids/contentassets/documents/unaidspublication/2011/JC2118 terminologyguidelines_fr.pdf. Consulté en ligne le 11/12/2013.

47 Barrère, P. «Jean-Luc Romero : "Je suis un survivant” 》. Le Dauphiné Libéré. 30 novembre 2012. http://www.ledauphine.com/sante/2012/11/30/jean-luc-romero-je-suis-un-survivant. Consulté en ligne le 11/12/2013.

48 INSERM. http://www.inserm.fr/thematiques/microbiologie-et-maladies-infectieuses/dossiers-d-information/sidavih. Consulté en ligne le 11/12/2013.

${ }^{49}$ Ibid.

${ }^{50}$ Sécoculture, sérodiagnostic, sérogroupe, sérolipase, séro-atténuation, séroneutralisation, séroprécipitation, pour n'en citer que quelques uns. Les termes « séropositif » et « séronégatif » apparaissent en 1980, soit plusieurs années avant la découverte du VIH, et servent à désigner un sujet dont le sérum contient des anticorps spécifiques , et non un malade du sida.

${ }^{51}$ James, D. « Afro, toxico et séropo ». Libération.fr. 12 mai 2003. http://www.liberation.fr/medias/2003/05/12/afrotoxico-et-seropo 433265. Consulté en ligne le 14/12/2013.

52 Lestrade D. "Sida : finalement, c'est plus dur d'être séropo en 2013 qu'il y a vingt ans ». Rue89.com. 13 septembre 2013. http://www.rue89.com/2013/09/13/finalement-cest-plus-dur-detre-seropo-2013-quil-y-a-vingt-ans245664. Consulté en ligne le 14/12/2013.

${ }^{53}$ de Beaulieu P. et M. «La femme séropo qui voulait avoir un enfant », in A votre Ecoute, coûte que coûte. france inter. $\quad$ http://www.franceinter.fr/emission-a-votre-ecoute-coute-que-coute-la-femme-seropo-qui-voulait-avoir-unenfant. Consulté le 14/12/2013. 
54 Favereau, E. «Sida : séropositifs, bien traités et non contaminants ». Libération, 10 mars 2014. http://www.liberation.fr/societe/2014/03/10/sida-seropositifs-bien-traites-et-non-contaminants 985972 .

${ }^{55}$ Environnement Canada, http://www.ec.gc.ca/eau-water/ . Consulté le 15/03/2014.

${ }^{56} \mathrm{NB}$ : Une portion de cette étude pourrait aussi porter sur les variations entre locuteurs français selon leur origine géographique, la terminologie du sida concernant tous les pays du monde francophone, tandis que l'épidémie ellemême est perçue de façon différente par chaque culture, ce qui ne manque pas de se refléter dans la terminologie propre à chaque région du monde. 